\title{
Travesía del financiamiento de la Comisión Nacional Para el Desarrollo de Pueblos y Comunidades indígenas a EMPRESAS DE TURISMO dE NATURALEZA eN MÉxICO
}

\author{
Bertha Palomino Villavicencio \\ berthapal13@gmail.com \\ Instituto Politécnico Nacional. ESCA Tepepan \\ Gustavo LóPez PARdo \\ Ipardo@unam.mx \\ Universidad Nacional Autónoma de México
}

\begin{abstract}
El turismo de naturaleza (TN) en México, al igual que en el resto del mundo, ha reportado en los últimos veinte años un incremento vertiginoso. Las instituciones mexicanas responsables de la política ambiental, de turismo y de desarrollo han sido protagonistas de este proceso, al haber incorporado al turismo de naturaleza en sus programas. En este trabajo, retomamos estudios realizados durante poco más de dos décadas a fin de conformar un panorama general de la política pública de impulso al tN en el país, en particular de la Comisión Nacional para el Desarrollo de los Pueblos y Comunidades indígena (CDI), como la agencia gubernamental de mayor protagonismo en este campo de acción, al canalizar miles de millones de pesos a esta actividad. Nuestra investigación tiene como objetivo analizar la intervención de esta dependencia a partir de cuatro momentos identificados en el desarrollo de su quehacer: sus objetivos, estrategias, reglas de operación, y resultados en cuanto a su financiamiento y distribución geográfica de los beneficios, lo que permitirá conocer a una escala general la actuación de la CDI en todo el periodo de su existencia, lo que servirá de base para el análisis de territorios concretos en los que se puedan identificar impactos, logros y tareas pendientes en las pretensiones de la política instrumentada.
\end{abstract}

Palabras clave: México, turismo de naturaleza, Comisión Nacional para el desarrollo de pueblos, comunidades indígenas, política pública.

\section{Journey of funds from the National Commission for the Development of Indigenous Communities to nature tourism COMPANIES In MeXico}

Nature Tourism has reported a rapid increase in the last twenty years in Mexico as in the rest of the world. The institutions dealing with environmental policy, development, and tourism have become the main actors in this process ever since they incorporated nature tourism in their programs. This work recovers the studies conducted in the last two decades to present a general overview of public policy that has boosted nature tourism in Mexico. The Comision Nacional para el Desarrollo de los Pueblos y Comunidades Indigenas (CDI) has been one of the most important governmental agencies participating in this field by channeling billions 
Travesía del financiamiento de la Comisión Nacional para el Desarrollo de Pueblos y Comunidades indígenas a empresas de turismo naturaleza en México

of pesos to this activity. Our research analyzes the participation of this agency from four identified moments within its activities -objectives, strategies, operation rules, and results in terms of its financing and geographical distribution of benefits. This will enable a concrete territorial analysis where we can identify the impact, achievements, and pending tasks in the implemented policy and its expectations.

Keywords: Mexico, National Commission for the development of towns, indigenous communities, nature tourism, public policy. 
n las últimas décadas, en todo el mundo, el turismo ha sido considerado un elemento estratégico para el desarrollo económico por su capacidad de generar divisas, empleos y estimular las economías regionales. Según la Organización Mundial del Turismo, en el 2017 se registraron más de 1323 millones de arribos turísticos que generaron alrededor de 1.34 billones de dólares americanos. La actividad aportó 10 \% del PIB mundial, representando $30 \%$ del valor de las exportaciones mundiales, y uno de cada 10 empleos fue generado en este sector (омт, 2018).

A finales del siglo xx, a raíz de la llamada crisis ambiental, en las sociedades industrializadas se vivieron transformaciones que marcaron modificaciones en las actividades económicas y sociales como respuesta a la búsqueda de una organización social y productiva que fuera más adecuada a la necesidad de la conservación de los recursos naturales y de la biodiversidad en general. En estas sociedades de detonó una conciencia ambiental y surgieron nuevos hábitos y estilos de vida ligados a lo natural y auténtico, en contraposición de lo artificial y cosificado, por lo que comenzó la demanda de nuevos destinos turísticos, mejor conservados y con actividades más participativas, diferentes a las ofrecidas por el modelo de sol y playa.

En este contexto de búsqueda del desarrollo sustentable, en el sector turístico se empezó a hacer referencia a formas de organización y prácticas turísticas diferentes como vía para la gestión de todos los recursos, de forma que pudieran satisfacerse las necesidades económicas, sociales y estéticas, respetando al mismo tiempo la integridad cultural, los procesos ecológicos esenciales, la diversidad biológica y los sistemas que sostienen la vida (омт, 1999). La sustentabilidad del turismo se volvió una condición, los territorios conservados y las culturas originarias y vivas se convirtieron en nuevos atractivos, del turismo en general y del turismo de naturaleza.

En México, el turismo también se incorpora en los planes nacionales de desarrollo y las distintas administraciones gubernamentales fijaron estrategias para mantener y aumentar su competitividad en el mercado internacional. Desde finales del siglo xx en la política turística mexicana se contempla la sustentabilidad como un componente central y se establece el fomento al Turismo de Naturaleza (TN) como un elemento para la diversificación de la oferta turística nacional.

En el 2005, la Secretaría de Turismo estableció como concepto de turismo de naturaleza: "Ios viajes que tienen como fin realizar actividades recreativas en contacto directo con la naturaleza y las expresiones culturales que le envuelven con una actitud y compromiso de conocer, respetar, disfrutar y participar en la conservación de los recursos naturales y culturales", abarcando las actividades de ecoturismo, turismo de aventura y turismo rural. A partir de ese año, las dependencias gubernamentales asumieron esta definición operacional y la incorporaron a sus programas institucionales (Castro y Fonseca, 2015, p. 189). Aunque después se hicieron precisiones al respecto en las propias dependencias gubernamentales, denominando también a este tipo de turismo como alternativo, turismo sustentable o, en forma más específica, ecoturismo; sin embargo solo para homogenizar este término se utilizará turismo de naturaleza para englobar las acciones desarrolladas en 
este tema por más de 15 instituciones gubernamentales en México.' Sin duda será una asignatura pendiente la revisión de estos conceptos y de sus sustratos teóricos, que no es la pretensión de este documento.

El tN se ha impulsado en México desde hace casi 25 años, principalmente por instituciones gubernamentales de los sectores económico, social y ambiental, la cuales, partiendo de la gran biodiversidad y la enorme riqueza cultural de nuestro país, han intentado utilizar este turismo como un detonador del desarrollo local y como respuesta a la agudización y profundización de la crisis del campo. En este proceso, las instituciones nacionales responsables de la política de desarrollo de los pueblos indígenas han tenido un papel central en la incorporación del ecoturismo como modalidad del tN en sus programas, con ese propósito han realizado acciones de apoyo y promoción a proyectos de este tipo en estas comunidades; primero con intervenciones aisladas y dentro de sus programas de producción regional o agroecológicos, y después como una línea estratégica de generación de capacidades y procesos productivos que permitieran mejorar sus condiciones de vida, fortalecer su organización social y modificar su tradicional vinculación desventajosa con el mercado nacional.

En este marco de participación de una amplia diversidad de organizaciones en el desarrollo del turismo de naturaleza, nos interesa hacer un viaje a través de los 15 años de intervención de la Comisión Nacional para el Desarrollo de los Pueblos y Comunidades indígenas (CDI), porque consideramos que tiene un papel protagónico y de liderazgo en este proceso. Tanto por el reto de abrirse y desarrollar esfuerzos para comprender y adaptarse, al igual que el campo mexicano a una nueva ruralidad -en particular las comunidades indígenas-, a una actividad productiva no tradicional de rápida transformación en el mercado; por el mayor financiamiento otorgado a empresas indígenas de turismo de naturaleza, así como por su dirección en las diferentes experiencias de colaboración interinstitucional que se han realizado.

De modo que este trabajo consiste en analizar la gestión de la CDI a través de las diversas acciones, proyectos y programas instrumentadas en relación con el apoyo al turismo de naturaleza en comunidades o grupos indígenas. Por tal motivo, se ha considerado en este trabajo la política pública como la serie de acciones de gobierno, resultado de diversas circunstancias políticas y demandas sociales. De tal suerte que es un conjunto de decisiones que se concretan en acciones orientadas a la realización de un objetivo para el beneficio público (Canto, 2002). Nuestro enfoque de análisis es el top-down (de arriba hacia abajo); o sea, desde el quehacer de las instituciones gubernamentales hacia la población directamente involucrada en el tema atendido para la ejecución de la política (Revuelta, 2007).

El desarrollo de acciones encaminadas a impulsar proyectos de turismo de naturaleza por los grupos indígenas por parte de la CDI obedece a una visión de alcanzar el mejora-

\footnotetext{
${ }^{1}$ Desde entonces, la información oficial sobre Turismo de Naturaleza incluye a los segmentos ecoturismo, turismo rural y turismo de aventura. Sectur (2006)
} 
miento de las condiciones de vida de estas comunidades, generando nuevas opciones económicas que aprovecharán la biodiversidad de sus territorios y el atractivo que ofrecen las culturas originarias al turismo moderno. El turismo era una nueva manera de integrar a los grupos indígenas a la modernidad, al tiempo que se revaloraba su cultura. En este sentido, la política de la CDI y las acciones para el fomento del tN se movían entre la tradición indigenista del Estado mexicano de integración-asimilación y los nuevos tiempos del reconocimiento a la multiculturalidad y reafirmación de lo diferente. En nuestro caso la acción de la CDI, al fomentar el turismo en estas comunidades, ha tenido los dos resultados; sin embargo, la explicación de esta cuestión será objeto de otro trabajo.

\section{Metodología}

Para esta investigación documental se hizo un estudio longitudinal retrospectivo del periodo 2000 al 2018, se retomaron estudios y productos previos generados en otras investigaciones por los autores de este documento, como la Base de Datos TurNatur. ${ }^{2}$

Se utilizaron las técnicas de análisis del discurso a diversas fuentes de información: documentos, reglamentos y lineamientos de operación de programas gubernamentales federales, relacionados con el desarrollo del tN en el país, para contar con un contexto general.

La atención se enfocó en la Comisión Nacional para el Desarrollo de los Pueblos y Comunidades Indígenas (CDI) como sujeto de estudio; se revisó la orientación de la política pública de la CDI para promover el desarrollo del turismo de naturaleza como actividad productiva en pueblos y comunidades indígenas; sus objetivos institucionales, sus estrategias de intervención; el financiamiento dedicado, además de sus resultados en cobertura y distribución geográfica. Las fuentes de información consultadas de esta gestión fueron los Proyectos y programas del Instituto Nacional Indigenista (INI), el Programa de Ecoturismo indígena (PEZI), el Programa de Turismo Alternativo en Zonas Indígena (PTAZI) y el Programa de Mejoramiento de la Producción y Productividad Indígena (PROIN); sus evaluaciones disponibles en el Consejo Nacional de Evaluación (Coneval), estudios realizados para la CDI, además de los informes proporcionados ex profeso por el Instituto Federal de Acceso a la Información (IFAI), órgano mexicano que garantiza el acceso a la información de las dependencias gubernamentales federales, así como libros y artículos publicados sobre el tema. Finalmente se realizó un tratamiento estadístico descriptivo de la información obtenida, el análisis y algunas reflexiones que abren también interrogantes a resolver.

\footnotetext{
${ }^{2}$ Base de Datos TurNatur construida en el marco del proyecto de investigación Las empresas comunitarias de turismo de naturaleza: factores de éxito y de fracaso, que con apoyo del fondo sectorial Sectur-Conacyt, realizamos en el Instituto de Investigaciones Económicas, de la Universidad Nacional Autónoma de México, en el periodo 2012-2014.
} 


\section{Desarrollo}

\subsection{Antecedentes}

Ante la crisis del campo que se vivió a finales de la década de los setenta, y en un contexto de crecimiento del llamado TN, el gobierno mexicano desarrolló una estrategia consistente en poner en valor los recursos culturales y naturales de las comunidades campesinas e indígenas, para su uso por el turismo. Durante más de 25 años distintas dependencias gubernamentales de los sectores económico, social y ambiental han intentado utilizar el TN como un diversificador de las opciones productivas en las zonas pobres, campesinas e indígenas, y como un detonador del desarrollo local, por lo cual han canalizado miles de millones de pesos para la conformación de proyectos y empresas que brindaran estas actividades y servicios turísticos en particular.

Aunque se han encontrado antecedentes de experiencias de emprendimientos de turismo de naturaleza en el país y de participación de diversas dependencias gubernamentales, las primeras experiencias de tN en su modalidad de proyectos de ecoturismo en las comunidades indígenas, ya identificados como tales, datan de 1989 bajo el impulso del proyecto Pueblos indígenas, ecología y producción para el desarrollo sustentable, en el Instituto Nacional Indigenista (INI) ${ }^{3}$ de la Secretaría de Desarrollo Social a nivel federal. Este proyecto se realizó en el marco del Programa de Agroecología Productiva con un carácter más bien marginal pero creciente en importancia, como lo demuestra las modificaciones paulatinas en la estructura institucional, el aumento de los beneficiarios y de los montos económicos dedicados a proyectos productivos del campo y de conservación ecológica, en donde apenas se dibuja el componente turístico.

Así, el Programa de Agroecología Productiva, con su modalidad de ecoturismo, avanza y asciende en importancia en el organigrama institucional, primero como un programa de la Subdirección de Salud y Bienestar Social de la Dirección de Operación y Desarrollo en 1995; después, en 1996 pasa a la Subdirección de Proyectos Especiales de la Dirección de Organización y Capacitación Social, se considera ya a los proyectos de ecoturismo como posibles detonadores desarrollo territorial a escalas comunitario, microrregional y regional sustentables, bajo el interés y la inserción consecuentes en las políticas públicas del país, de la importancia internacional de lo ambiental y de la propuesta del desarrollo con sustentabildad. A partir de entonces, se inicia la coordinación interinstitucional con otras

\footnotetext{
${ }^{3}$ www.cdi.gob.mx/ini/. Programas y Proyectos de Instituto Nacional Indigenista. Dirección de Operación y Desarrollo., México, 2003. Desde la creación del Instituto Nacional Indigenista (INI), en 1948, se reconoció que esta población debería tener una atención especial del Estado, debido a las condiciones de marginación y discriminación en que vivían. A partir de este reconocimiento se impulsaron políticas públicas, en materia de desarrollo económico, educación, salud, cultura y justicia, fundamentalmente de tipo asistencial, que tenían como objetivo ayudar a que disminuyeran las condiciones de pobreza y marginación en que se encontraban. En el Plan Nacional de Desarrollo de los Pueblos Indígenas 1991-1994 se contempló el apoyo a iniciativas para el diseño y operación de programas productivos y de conservación de la biodiversidad, acordes a las necesidades e intereses de las poblaciones indígenas locales; y la exploración de perspectivas de desarrollo que combinaran adecuadamente los recursos y los conocimientos propios de los pueblos indígenas con ofertas externas disponibles, conforme a su propia elección, para lograr programas de desarrollo rural y regional culturalmente apropiados y ecológicamente sustentable.
} 
dependencias, principalmente vinculadas con el ámbito ambiental y de desarrollo social, buscando la sinergia en sus acciones y resultados.

En 1999, el Programa de Agroecología Productiva se incorpora a la Subdirección Operativa de Fondos Regionales, de la Dirección de Operación y Desarrollo del INI, un area (o el área) de mayor importancia en esta institución, con lo cual este programa adquiere un mayor protagonismo.

En el periodo de 1995 a 1999 el INI destinó 9.6 millones de pesos para proyectos productivos sustentables, incluido el ecoturismo, y de una atención inicial de 8 se pasó a 106 proyectos (Palomino y López, 2005).

\section{2. La Comisión Nacional para el Desarrollo de los Pueblos y Comunidades Indígenas en el escenario del turismo de naturaleza}

En la gestión de la CDI se identifican tres grandes etapas de promoción y apoyo de turismo de naturaleza como opción productiva en esta población objetivo.

\subsubsection{Primera etapa. Transición institucional IN//CDI del 2000 al 2005}

En el marco del inicio del nuevo milenio, con una gran efervescencia mundial por el turismo alternativo y de la demanda internacional por nuevos destinos con patrimonios naturales y culturales conservados, el gobierno federal, algunos gobiernos estatales, municipales y organizaciones de la sociedad civil, entre los años 2000 al 2005, destinaron 1465.31 millones de pesos para el fomento y desarrollo del turismo de naturaleza, dando por creados 1239 emprendimientos. Como expresión de este interés, en ese periodo se conformó el primer grupo interinstitucional de turismo de naturaleza que busco la coordinación de 10 dependencias gubernamentales que de diversas maneras apoyaban el tN en México. Este grupo Interinstitucional elaboró el primer inventario nacional de empresas y proyectos ecoturísticos, con la información que cada una de las dependencias facilitó sobre los proyectos apoyados de 2001 a 2005, en donde ya se empezaba a destacar la participación de los proyectos impulsados por grupos y comunidades indígenas (Sectur, 2006).

En particular, el INI en los primeros años de esta etapa, y después la cDI, fortalecieron el apoyo a proyectos ecoturísticos desde un enfoque fundamentalmente conservacionista, sobre todo en áreas naturales protegidas (ANP).

En el año 2001, el ecoturismo se ubicaba aún dentro de la estructura normativa del INI, vinculado a proyectos de agroecología productiva, dentro del Programa de Desarrollo Económico Productivo, en la Subdirección de Fondos Regionales. Pero para el 2003 alcanzó el rubro de modalidad en el Programa de Impulso a Proyectos Sustentables en Zonas Indígenas, que englobó a todos los proyectos considerados de agroecología productiva, de conservación y de turismo.

A mediados del año 2003 se crea la Comisión Nacional para el Desarrollo de los Pueblos y Comunidades Indígenas (CDI), como organismo descentralizado, con autonomía presupuestal, operativa, técnica y administrativa, que se conforma en una fusión complicada entre 
el Instituto Nacional Indigenista (INI), la Coordinación General del Plan Nacional de Zonas Deprimidas y Grupos Marginados (Coplamar) y algunos sectores de la Secretaría de Desarrollo Social (Sedesol). Desde entonces, esta dependencia es la responsable principal de dirigir, instrumentar, coordinar y evaluar, entre otras atribuciones, la actuación del gobierno federal, relacionada con la población indígena, basada en principios de transversalidad, integridad, sustentabilidad y consulta a las poblaciones indígenas. Sin embargo, durante el periodo transicional de esta novedosa y sui generis dependencia, en ese momento de la historia de la administración pública, los programas y proyectos del INI a la CDI se mantuvieron operando bajo la misma inercia.

Del 2001 al 2005 se canalizaron poco más de \$ 165 millones de pesos a esta actividad, a través de 380 acciones $^{4}$ que involucraron a 39742 indígenas en 23 entidades del país.

Cuadro 1. Inversión anual del Instituto Nacional Indigenista y la Comisión Nacional para el Desarrollo de los Pueblos y Comunidades Indígenas 2000-2005

\begin{tabular}{llccc}
\hline Institución & Programa & Año & Acciones & Monto (\$) \\
\hline $\begin{array}{l}\text { Instituto Nacional } \\
\text { Indigenista }\end{array}$ & Criterio de elegibilidad & 2000 & 65 & 29567289.94 \\
& agroecología productiva & 2001 & 114 & 37366114.00 \\
& & 2002 & 46 & 22439435.00 \\
\hline INI /CDI & $\begin{array}{l}\text { Programa Impulso a Proyectos } \\
\text { SDI }\end{array}$ & 2003 & 47 & 18576779.00 \\
& Sustentables en Zonas Indígenas & & & \\
& $\begin{array}{l}\text { Programa Impulso a Proyectos } \\
\text { Sustentables en Zonas Indígenas }\end{array}$ & 2004 & 39 & 18864484.00 \\
\hline Total & $\begin{array}{l}\text { Programa de Ecoturismo en Zonas } \\
\text { Indígenas }\end{array}$ & 2005 & 69 & 38405008.00 \\
\hline
\end{tabular}

Fuente: Elaboración propia, 2019.

No obstante este incremento de los recursos, y aunque había una convicción, por lo menos discursiva del cuerpo directivo de la CDI de que el "ecoturismo" representaba para algunas comunidades la mejor y, en ocasiones, la única opción para el desarrollo, tampoco la nueva administración elaboró una propuesta programática que señalara el rumbo, los objetivos, las metas, las estrategias y las acciones del entonces llamado ecoturismo indígena. Al igual

${ }^{4}$ En los registros de resultados de los programas de la CDI analizados en todo este trabajo, existe como una constante el reporte del número de acciones desarrolladas y no siempre el número de proyectos apoyados, por lo que este documento hará referencia a este indicador. Cabe mencionar que un proyecto o empresa de turismo de naturaleza apoyado puede haber sido beneficiado con una o más acciones en el mismo año. 
que el INI, la CDI impulsó en esta primera etapa acciones (dinero, materiales y capacitación) para el desarrollo de estas actividades sin tener una claridad, no solo del rumbo a seguir, sino de las necesidades específicas para consolidar los proyectos y alcanzar el éxito que permitiera el cumplimiento de los objetivos institucionales.

En esta etapa, el tratamiento marginal de las actividades turísticas denominadas genéricamente como ecoturismo reflejo con claridad la indefinición que tuvo el INI y la cDI, en sus primeros años, sobre esta actividad, pues aunque en el discurso a partir del 2002, en el marco del Año Internacional del Ecoturismo, se le asignaba un papel importante en la estrategia para el desarrollo social y económico de las comunidades indígenas, en la práctica no se aplicaron los recursos financieros ni se creó la estructura operacional correspondiente a tal significación. Primero en el INI y después en la CDI, el financiamiento para el desarrollo de actividades turísticas a cargo de las comunidades indígenas era reducido, no contaban con una estructura institucional definida, estuvieron durante todo el periodo, dependientes de la dinámica y bajo la dirección del gran programa Fondos Regionales; pero sobre todo los operadores institucionales, acostumbrados a la producción primaria y secundaria del campo mexicano, no solo no comprendían del todo la complejidad del turismo, como una actividad económica proveedora de servicios, sino que además se confundían actividades de turismo rural o de aventura con las de ecoturismo (López y Palomino, 2019).

La realidad de la intervención del INI/CDI en este periodo fue presentada en la Evaluación de Resultados 2001-2004 del Proyecto Ecoturismo, realizada por unAm en el año 2005, que mostró las debilidades institucionales en cuanto a la instrumentación y evaluación de su quehacer. Pero lo más revelador fue el hallazgo de que muy pocos proyectos estaban en operación y enfrentaban una serie de problemas organizativos, financieros, técnicos y de comercialización que impedían su consolidación.

\subsubsection{Segunda etapa: Institucionalización y auge del turismo de naturaleza, 2006-2012}

Desde el Programa Sectorial de Turismo 2007-2012 (Sectur, 2008) se estableció el fortalecimiento económico del turismo con una orientación hacia la sustentabilidad. (Presidencia de la República, 2006). En esta estrategia, el turismo de naturaleza adquirió especial relevancia no solo para este sector gubernamental, sino para otros ámbitos gubernamentales relacionados con el mejoramiento de las condiciones de vida de la sociedad, el combate a la pobreza y la conservación del ambiente (López, 2012). Así, durante este periodo, 16 dependencias federales, incluida la CDI, tenían signado en sus objetivos institucionales y en sus documentos normativos el apoyo al TN a través de 42 programas de acción, y destinaron entre todas ellas 3181314070 pesos para el desarrollo de esta actividad (López y Palomino, 2014).

En la CDI, por fin en el año 2006, las actividades de turismo de naturaleza adquieren la categoría de programa institucional como Programa de Ecoturismo en Zonas Indígenas (PEZI), con reglas de operación claras y situado en una de las áreas institucionales de mayor importancia de la propia CDI, la Coordinación General de Programas y Proyectos 
Especiales. El objetivo general de este programa fue: “Contribuir al desarrollo de la población indígena mediante la ejecución de acciones en materia de ecoturismo, aprovechando el potencial existente en las regiones indígenas, otorgando apoyos para elaborar y ejecutar proyectos encaminados al aprovechamiento sustentable de sus bellezas naturales y patrimonio cultural” (CDI, 2006, p.13), con una atención focalizada a núcleos agrarios, organizaciones y grupos de trabajo de 871 municipios indígenas prioritarios por su nivel de pobreza, a diferencia de los proyectos y programas previos del INI y la CDI, que tenían como población objetivo a toda la población indígena del país.

Los tipos y montos de apoyo del PEZI que se ofertaron en orden de mayor a menor importancia económica fueron: la construcción de instalaciones y equipamiento, la elaboración de proyectos, estudios y permisos, formación, fortalecimiento, difusión, seguimiento y evaluación, en rangos de \$1500 000 a \$50 000, fortaleciendo así una visión tradicional del turismo que prioriza las instalaciones sobre la planeación del negocio, el desarrollo de capacidades, la comercialización, pero sobre todo el diseño de productos turísticos (Palomino y López, 2007).

Un año después, en el 2007, el PEZI se convierte en el Programa de Turismo Alternativo en Zonas indígenas (PTAZI) que llegaría para quedarse durante el resto de este periodo analizado. Algunos antecedentes de esta transformación fueron los resultados y las recomendaciones de la Evaluación de Resultados 2001-2004 del Proyecto de Ecoturismo, que concluyó que los proyectos financiados en este programa correspondían más a diversas modalidades de turismo alternativo y mucho menos a ecoturismo, por lo que se decidió cambiar y/o adaptar el programa a esta realidad, empezando por cambiar el nombre del proyecto; además de dirigir esta actividad a valorar económica y socialmente los patrimonios natural y cultural de las comunidades en que se desarrollarían los proyectos de turismo de naturaleza, con el objeto de incidir en las condiciones de vida de la población vinculada a estos emprendimientos.

De esta manera el objetivo general del PTAZl fue impulsar el desarrollo de los pueblos indígenas mediante el apoyo de proyectos de turismo alternativo (también llamado de naturaleza), poniendo en valor su patrimonio natural y cultural desde una perspectiva sustentable a fin de mejorar la calidad de vida, en un principio a partir del aumento de los ingresos de esta población. Para ello se promovía la adquisición de capacidades de los participantes directos y de las comunidades en las que se llevaban a cabo estas actividades, para apoyar sus procesos organizativos y coadyuvar a mejorar la calidad de los productos y servicios turísticos ofrecidos, con un interés particular por incorporar a las mujeres en todo el proceso del proyecto, con responsabilidades y beneficios. Así como la puesta en marcha de estrategias para la difusión y promoción de los proyectos.

Por eso el PTAZI proporcionaba financiamiento para la elaboración, ejecución y promoción de proyectos; plan de negocios, estudios técnicos y pago de permisos; para la dotación de infraestructura y equipamiento, formación, fortalecimiento organizativo comunitario y empresarial, la difusión y promoción. Alcanzando montos superiores de los dos millones de pesos para la consolidación de estas empresas, a partir de un proceso más 
Cuadro 2. Inversión anual de la Comisión Nacional para el Desarrollo de los Pueblos y Comunidades Indígenas (CDI) en turismo de naturaleza, 2006-2012

\begin{tabular}{|c|c|c|c|c|c|}
\hline Institución & Programa & Siglas & Año & Acciones & Monto (\$) \\
\hline \multirow{8}{*}{$\begin{array}{l}\text { Comisión } \\
\text { Nacional para } \\
\text { el Desarrollo de } \\
\text { los Pueblos y } \\
\text { Comunidades } \\
\text { Indígenas }\end{array}$} & $\begin{array}{l}\text { Programa de Ecoturismo en } \\
\text { Zonas Indígenas }\end{array}$ & PEZI & 2006 & 154 & 118156679.67 \\
\hline & \multirow{6}{*}{$\begin{array}{l}\text { Programa de Turismo } \\
\text { Alternativo en Zonas Indíge- } \\
\text { nas Zonas Indígenas }\end{array}$} & \multirow[t]{6}{*}{ PTAZI } & 2007 & 170 & 129072148.17 \\
\hline & & & 2008 & 162 & 146663864.00 \\
\hline & & & 2009 & 151 & 146250000.00 \\
\hline & & & 2010 & 194 & 155870079.78 \\
\hline & & & 2011 & 307 & 188423399.83 \\
\hline & & & 2012 & 255 & 170229522.82 \\
\hline & & & & 1393 & 1054665694.27 \\
\hline
\end{tabular}

Fuente. Elaboración propia, 2019.

selectivo de financiamiento, pero aunque se diversificaron los rubros de los apoyos, la tendencia continuó hacia la priorización de la planta turística (CDI, 2007).

A través de los siguientes años, el énfasis de los apoyos a las empresas de turismo de naturaleza fue trasladándose paulatinamente hacia la evaluación de la viabilidad económica de los proyectos; fortalecimiento de capacidades, comercialización y marketing, pero también de la organización empresarial y comunitaria; así como al apoyo de figuras de asociatividad, como las empresas integradoras que pudieran tener mayores impacto en la competitividad en el mercado a partir del desarrollo de cadenas de valor. Lo anterior con la intención de contribuir al desarrollo económico de la población indígena a través del aprovechamiento sustentable de los recursos naturales de las zonas donde habitan y de su patrimonio cultural. Así, este programa otorga subsidios a núcleos agrarios, organizaciones y grupos de trabajo conformados por indígenas, para el establecimiento y desarrollo de sitios de turismo alternativo, principalmente en su vertiente de turismo de naturaleza.

El periodo entre el 2006 y el 2012 fue una etapa de precisión en las estrategias de intervención de la cDI, pues aunque se mantenía la política de distribución de recursos a los grupos indígenas bajo la lógica de mitigar la pobreza, también se reconoció que el éxito de la actividad no solo dependía de contar con recursos naturales y culturales, sino que era indispensable que los productos y servicios que en materia de turismo ofertaran los pueblos, comunidades y grupos indígenas fueran de calidad. Por ello, cada vez más se intentaba mejorar las capacidades de los grupos, familias y comunidades para adquirir nuevas habilidades que permitieran dar un mejor servicio, y en menor medida el fortalecimiento de la organización social comunitaria.

La CDI destinó los mayores apoyos financieros al número más grande de acciones para proyectos o empresas de turismo de naturaleza en comunidades indígenas, así como el porcentaje más alto en relación con su presupuesto total; y el Programa Turismo Alternativo en Zonas Indígenas (PTAZI) canalizó \$ 1054665694.27 (cuadro 2), un poco más de 33 \% 
del monto financiando por la CDı para el tN durante los 15 años de su funcionamiento, en beneficio de 56432 personas (38 452 hombres y 17980 mujeres) (López y Palomino, 2014).

\subsubsection{Tercera etapa. Apoyo selectivo a empresas de turismo de naturaleza con mayor potencialidad, del 2013 al 2018}

Esta etapa corresponde a un nuevo gobierno en el país, en el que la CDI mantiene el rol principal en cuanto a la atención a la población indígena y a sus proyectos productivos, como el TN, pero con modificaciones en la estructura y orientación de su gestión.

De esta manera la CDI se reestructura en varias coordinaciones para fortalecer programas que se consideraron vinculados y vinculantes con la pretensión de que en sinergia obtuvieran mejores resultados. Una de ellas, la Coordinación General de Fomento al Desarrollo Indígena (CGFDI) crea en el año 2014 el Programa para el Mejoramiento de la Producción y la Productividad Indígena (Proin) como resultado de la fusión de seis programas previos: Programa para el Apoyo a la Producción Indígena, Programa de Turismo Alternativo en Zonas Indígenas, Programa Organización Productiva para Mujeres Indígenas, Programa Fondos Regionales Indígenas, Proyectos de Manejo y Conservación de Recursos Naturales en Zonas Indígenas y Proyecto Fortalecimiento de Capacidades para los Indígenas (Coneval-Proin, 2015, p.5).

El objetivo general del Proin fue "impulsar la consolidación de proyectos productivos y turísticos, así como de acciones de seguridad alimentaria para la población indígenas organizada en grupos, sociedades o empresas que habitan en localidades con $40 \%$ y más de población indígena, para mejorar sus ingresos monetarios y no monetarios y crear condiciones de igualdad entre las mujeres y los hombres" (INPI, 2019, p.1). Con esto la población objetivo del Proin se reduce y concreta de una población total indígena de 5841427 personas, a una población objetivo 224536 personas (Coneval, 2017, p .8)

El marco directo de política pública del Proin fue el Programa Especial de Pueblos Indígenas (PEPI) 2014-2018, con su objetivo: "Mejorar el ingreso monetario y no monetario de la población indígena a través del impulso a proyectos productivos"; $y$ en forma indirecta con la meta II, México incluyente del Plan Nacional de Desarrollo (PND) 2013-2018, en su objetivo 2.2 que consistía en: "Transitar hacia una sociedad equitativa e incluyente", a través de la estrategia de: "Fomentar el bienestar de los pueblos y comunidades indígenas, fortaleciendo su proceso de desarrollo social y económico, respetando las manifestaciones de su cultura y el ejercicio de sus derechos".

Los apoyos a proyectos productivos otorgados por el Proin fueron para las siguientes modalidades: Mujer indígena (MI); Proyectos Productivos Comunitarios (PPC); Turismo de naturaleza; y Apoyos complementarios para el desarrollo indígena, como Acciones para la adaptación y mitigación de los efectos de cambio climático, Comercialización, o Capacitación, asistencia técnica, acompañamiento y certificación a proyectos de productores indígenas, entre otros.

La conformación del Proin como gran programa "paraguas" coordinador para el impulso y apoyo a las actividades productivas desarrolladas por su población objetivo, fue 
el diagnóstico de la gestión de la CDI en su vertiente productiva, elaborado por la propia institución, destacando entre otros aspectos:

Problemas: 1) Ausencia de capacitación técnica, empresarial y estratégica para cada proyecto; 2) Los productores tienen insuficiente promoción comercial de sus productos; 3) Inadecuada asistencia técnica; 4) Los proyectos productivos de la población indígena no son aprobados para su apoyo y 5) Los proyectos productivos aprobados no inician operaciones.

Efectos de la existencia de los problemas: 1) Organizaciones sin acceso al financiamiento de los agentes productivos, 2) Finanzas débiles de la empresa; 3) Alta presencia de intermediarismo comercial; 4) Organizaciones muy propensas al fracaso y 5) Escasa innovación (CDI, 2014, p.17-19)

Además del panorama anterior, también la nueva administración de la CDI contó en particular con los diagnósticos realizados por académicos y consultores sobre las empresas de indígenas de turismo de naturaleza que fortalecieron el cambio de orientación en la modalidad de turismo en el Proin:

- La última evaluación de desempeño del PTAZı en 2009, que marcaba debilidades en cuanto el logro de indicadores; problemas de diseño; de evaluación y seguimiento de beneficiarios e impacto; además de ausencia o ineficiencia de programas de promoción comercial de las empresas financiadas; hallazgos nuevamente preocupantes, a pesar de estrategias dirigidas al fortalecimiento gerencial de las empresas indígenas de turismo de naturaleza apoyadas con dictámenes previos a la aprobación de apoyos por la propia Secretaría de Turismo o de dependencias económicas y ambientales. (Coneval, 2009).

- El estudio diagnóstico de 167 empresas turísticas indígenas que realizó la consultora Vamos de Puente, en el año 2010 (VDP, 2010) por encargo ex profeso de la CDI, dando resultados "estremecedores en cuanto a la ineficacia de este programa al lograr realmente que muy pocos de los centros turísticos estén funcionando y logrando atraer turistas, y por tanto mejorar realmente las condiciones de vida de las poblaciones indígenas" (Ávila, 2015, p. 15).

- La investigación Las empresas comunitarias de turismo de naturaleza: factores de éxito $y$ de fracaso del Fondo sectorial Sectur-Conacyt, desarrollada por la UnAm y presentada a la CDI, que identificó en un universo de más de 2000 empresas de turismo de naturaleza a 998 organizadas por comunidades indígenas, de las cuales solo se destacaron 32 por contar con una serie de indicadores de éxito potencial en el mercado de este segmento turístico (López y Palomino, 2014). A este grupo de empresas se les denominó "Empresas Bandera” para que las instituciones enfocarán sus programas y acciones al fortalecimiento de estas experiencias productivas, con el objeto de obtener mayores impactos de sus políticas públicas, así como la conformación a mediano plazo de un grupo de empresas competitivas y exitosas desde una perspectiva de rendimientos económicos, beneficios sociales y ambientales para su entorno social. Este grupo de empresas destacadas por un "efecto espejo" impulsaría el desarrollo de otras experiencias productivas más rezagadas de turismo de naturaleza en las comunidades indígenas. 
En consecuencia, en esta etapa observamos un cambio en la actuación de la CDI, particularmente en lo referente a buscar el acompañamiento de las empresas y proyectos indígenas de turismo, a fin de apoyar la consolidación de los emprendimientos más exitosos, reforzar los que aún se encontraban en una situación intermedia o inicial y solo emprender nuevos proyectos, previa realización de los estudios o planes de negocios. En esta etapa la Comisión le apuesta a fortalecer las capacidades ya existentes, pero también a impulsar las actividades de difusión, promoción y desarrollo de estrategias de comercialización, fundamentales para el posicionamiento de los proyectos ecoturísticos. Se trataba de contribuir a la consolidación de la comercialización de los productos y servicios de TN más posicionados en el mercado. La estrategia fue la creación en el año 2015 de la marca Paraísos Indígenas, que sirviera de plataforma a todas las empresas indígenas, para lo cual había que buscar cierta estandarización en la calidad de los servicios, en el cumplimiento de los requisitos de las buenas prácticas ambientales propias de las empresas de turismo de naturaleza y en una mayor vinculación con la economía regional (INPI, 2019). Estas empresas deberían contar con sellos y distintivos nacionales e internacionales, como el de la Norma Oficial Mexicana NOM-133- SEMARNAT-2015, que regula las instalaciones y/o actividades para que sean amigables con el medio ambiente; el distintivo "M" de buenas prácticas empresariales o el" Punto limpio" que son otorgados por la Secretaría de Turismo federal.

El Programa Paraísos Indígenas es resultado de la coordinación interinstitucional encabezada por la CDI con la participación de la Secretaría de Turismo (Sectur), la Secretaría del Medio Ambiente y Recursos Naturales (Semarnat) y la Financiera Nacional de Desarrollo Agropecuario, Rural, Forestal y Pesquero (INPI, 2019). Aglutina a 105 sitios turísticos de alto valor natural y cultural en manos de las comunidades indígenas que ofertan servicios y actividades de ecoturismo, turismo rural, de aventura y de salud y bienestar. Las empresas que conforman los Paraísos Indígenas (entre ellas las 32 identificadas como empresas bandera), no solo cuentan con infraestructura para brindar actividades y servicios con calidad, sino que además son operadas por indígenas capacitados para realizar las actividades que ofertan, ya de aventura, senderismo, ciclismo, avistamiento de flora y fauna, entre otras (INPI, 2019).

Paraísos indígenas se conformó como una marca que respaldaba y garantizaba cierta calidad en el servicio y las actividades turísticas, que además de darle certidumbre a los turistas independientes que buscan nuevos destinos y experiencias cercanos a la naturaleza y a culturas anfitrionas, también servirá de base para concretar una serie de alianzas comerciales con los operadores turísticos, nacionales y extranjeros. ${ }^{5}$

Con Paraísos indígenas la CDI también incrementó su participación en ferias turísticas especializadas de turismo de naturaleza, incluso ganó premios al mejor producto turístico alternativo, como en el caso de la XXIII edición de la Feria Internacional de Turismo (Fitur) desarrollada en enero de 2018 en Madrid, España, en donde obtuvo, junto con la Revista Aire Libre, el premio como el "Mejor producto de turismo activo, en su categoría internacional.

${ }^{5}$ Booking, Tours and Travels, Expedia, Tripadvisor, Adventur México, Travel, Zona Turística, Guía México, Pricel Traver, Viajes Bojórquez, entre otras. 
De acuerdo con los datos oficiales, esta estrategia permitió generar cinco mil empleos directos y favorecía que alrededor de 700000 personas, promedio al año, visitaran estos destinos (INPI, 2019).

En general, del 2013 al 2018, la vertiente de turismo de naturaleza del Proin destinó un poco más de 800 millones de pesos para desarrollar 1017 acciones de apoyo a empresas indígenas de esta actividad productiva.

Cuadro 3. Inversión anual de la Comisión Nacional para el Desarrollo de los Pueblos y Comunidades Indígenas en turismo de naturaleza, 2013-2018

\begin{tabular}{|c|c|c|c|c|c|}
\hline Institución & Programa & Siglas & Año & Acciones & Monto \\
\hline \multirow{7}{*}{$\begin{array}{l}\text { Comisión } \\
\text { Nacional para } \\
\text { el Desarrollo de } \\
\text { los Pueblos y } \\
\text { Comunidades } \\
\text { Indígenas }\end{array}$} & $\begin{array}{l}\text { Programa para Mejoramiento } \\
\text { de la Producción y } \\
\text { Productividad Indígena }\end{array}$ & Proin & 2013 & 237 & 169102520.47 \\
\hline & & & 2014 & 241 & 175268030.33 \\
\hline & & & 2015 & 208 & 216896223.85 \\
\hline & & & 2016 & 221 & 200319337.20 \\
\hline & & & 2017 & 49 & 22100000.00 \\
\hline & & & 2018 & 61 & 34000000.00 \\
\hline & & & Total & 1017 & 817686111.85 \\
\hline
\end{tabular}

Fuente: Elaboración propia, 2019.

Como colofón de este recorrido por 15 años de la CDI decidimos presentar algunos datos para contar con un escenario más objetivo de la importancia y trascendencia de los diversos programas de turismo desarrollados por esta institución.

Aunque nuestros datos sobre el financiamiento que todas las dependencias gubernamentales han canalizado al fomento del TN solo llegan hasta el 2017, si nos pueden dar una idea de la importancia para esta actividad de la acción de la CDI, pues de los 7557200340 pesos que durante el periodo 2000-2017 se destinaron al fomento del tN, 26.76 \% lo aportaron programas del INI/CDI.

Ese financiamiento se distribuyó a 26 entidades del país; Oaxaca, Chiapas, Hidalgo, Michoacán, Yucatán y Veracruz concentraron 61 \% de esta cantidad (véase figura 1). Esta concentración es congruente con el enfoque territorial de las políticas nacionales de combate a la pobreza y de conservación de los recursos naturales; así, con la mayor presencia de población indígena en estas entidades, debido al reconocimiento de ellas, existe una gran biodiversidad y/o cuentan con culturas originarias de gran vitalidad. 
Travesía del financiamiento de la Comisión Nacional para el Desarrollo de Pueblos

y Comunidades indígenas a empresas de turismo naturaleza en México

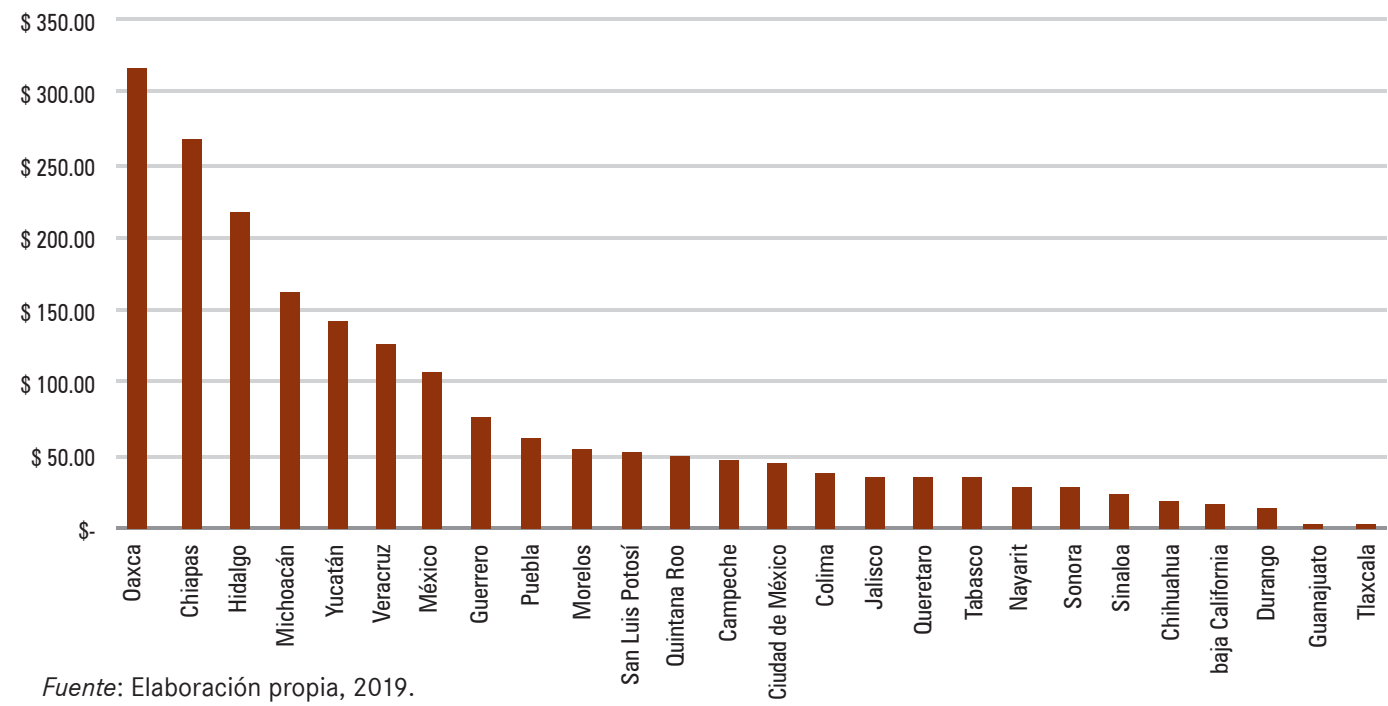

Figura 1. Inversión total de la CDI en turismo de naturaleza por entidad federativa 2000-2018 (millones de pesos)

\section{A manera de conclusión}

Como se deriva de los datos, en México un protagonista del desarrollo del tN es la CDI, es esta institución la que más ha invertido para el desarrollo de las actividades turísticas entre las comunidades y pueblos indígenas, desde la perspectiva de aprovechar el boom del Ilamado turismo de naturaleza para mejorar sus condiciones de vida. No obstante, durante los años que operó esta institución careció de una orientación programática; es decir, un instrumento rector, un programa que estableciera los objetivos, acciones, metas de dichas acciones, que definiera el tipo de turismo que era más adecuado a las comunidades indígenas, que reflejara sus intereses y que los capacitara para aprovechar la inversión gubernamental y construir opciones de desarrollo de sus comunidades. Su ausencia se refleja en la orientación centrada en la formación de infraestructura y equipamiento para la realización de actividades y servicios, sin atender el fortalecimiento de las capacidades de los grupos que se involucraron en dicha actividad, y fue determinante para que se conformara un sector del turismo de naturaleza indígena sin condiciones para competir adecuadamente en el mercado turístico y desvinculado en su mayoría de la actividad turística nacional

Las acciones de la CDI obedecieron a final de cuentas a sus objetivos institucionales de combate a la pobreza y solo al final de su existencia procuró acciones más encaminadas a fortalecer los proyectos indígenas, dotándolos de mecanismos que les ayudaran a competir en el mercado del turismo de naturaleza. La efectividad de dicha acción está por verse. No es suficiente realizar evaluaciones de diseño o de desempeño, sobre los asuntos administrativos de los programas, es necesario realizar por primera vez una evaluación del impacto en las comunidades y grupos beneficiados, si generaron capacidades y pudieron detonar procesos de economía local. 


\section{Referencias}

Ávila Romero, A. (2015). Análisis del turismo alternativo en comunidades indígenas de Chiapas, México, Études caribéennes , 31-32. Disponible en http://journals.openedition.org/etudescaribeennes/7601

Canto, M., (2002). Introducción a las políticas públicas. En M. Canto Chac (Coord.), Participación ciudadana y políticas públicas en el municipio. México: Ediciones del Movimiento Ciudadano por la Democracia.

Castro Álvarez, U. y Fonseca Morales, M. A. (2015). Turismo alternativo y políticas públicas en México. En M. Velarde Valdez, A.V. C. Maldonado Alcudia y S. Gómez Nieves. (Coord), Referentes teóricos del turismo alternativo. Enfoque en comunidades rurales (pp. 183-218). Serie Ciclos y Tendencias en el Desarrollo de México/43. Guadalajara, Los Mochis, Los Ángeles: Universidad de Guadalajara/Universidad de Occidente/ UCLA Program on México Profmex/ Juan Pablos Editores.

Comisión Nacional para el Desarrollo de los Pueblos Indígenas (2003). Programas y Proyectos de Instituto Nacional Indigenista. México: Dirección de Operación y Desarrollo, Autor. Disponible en: www.cdi.gob.mx/ini/.

Comisión Nacional para el Desarrollo de los Pueblos Indígenas (2006). Programa de Ecoturismo en Zonas Indígenas, México: Autor

Comisión Nacional para el Desarrollo de los Pueblos Indígenas (2007). Programa de Turismo Alternativo en Zonas Indígenas. México: Autor. Disponible en: www.cdi.gob.mx

Comisión Nacional para el Desarrollo de los Pueblos Indígenas (2014). Diagnóstico del Programa para el Mejoramiento de la Producción y Productividad Indígena. Documento interno.

Consejo Nacional de Evaluación de la Política de Desarrollo Social (2009). Informe de la Evaluación Específica de Desempeño PTAzı 2009-2010. México: Autor.

Consejo Nacional de Evaluación de la Política de Desarrollo Social (2015). Informe de la Evaluación del Programa para el Mejoramiento de la Producción y la Productividad Indígena (Proin). México.

Consejo Nacional de Evaluación de la Política de Desarrollo Social (2017). Informe de la Evaluación del Programa para el Mejoramiento de la Producción y la Productividad Indígena (Proin). México

INPI (2019). https://www.gob.mx/inpi, consultado 20 mayo 2019.

López, G. (2012). Políticas gubernamentales para el desarrollo del turismo de naturaleza en comunidades y pueblos indígenas de México. Cuadernos de Patrimonio Cultural y Turismo, 19, pp. 102-109.

López, G. y Palomino, B. (2014). El turismo naturaleza en comunidades y pueblos indígenas. Informe técnico de la investigación "Las empresas comunitarias de turismo de naturaleza: factores de éxito y de fracaso". Fondo sectorial Sectur-Conacyt. México: IIEC-UNAM.

López, G. y Palomino, B. (2019). Turismo de naturaleza en comunidades indígenas en México. México. IIEc-unam. 
Travesía del financiamiento de la Comisión Nacional para el Desarrollo de Pueblos y Comunidades indígenas a empresas de turismo naturaleza en México

Organización Mundial de Turismo (1999). Guía para las administraciones locales: Desarrollo turístico sostenible. Madrid: Autor.

Organización Mundial de Turismo (junio de 2018). Barómetro de turismo. Madrid, España: Autor .

Palomino, B. y López, G. (2005). Evaluación de resultados 2001-2004 del Proyecto de Ecoturismo. Informe Final. CDI. México: unAm/CDI.

Palomino, B. y López, G. (2007). Evaluación 2006 del Programa de Ecoturismo en Zonas Indígenas. México: $\mathrm{CDI}$.

Presidencia de la República (2006). Plan Nacional de Desarrollo 2007-2012. Disponible en http://pnd.calderon.presidencia.gob.mx/economia-competitiva-y-generadora-de-empleos/turismo.html

Programa de las Naciones Unidas para el Desarrollo (2014). Diagnóstico Complementario sobre Aspectos Específicos para el Diseño del "Programa para el Mejoramiento de la Producción y Productividad Indígena. Estudios. Documento interno

Revuelta Vaquero, B. (2007). La implementación de políticas públicas. Díkaion. 21(16), 135-156

Secretaría de Turismo (2006). El Plan Estratégico de Turismo de Naturaleza. 2006-2015. México: Xerox.

Secretaría de Turismo (18 de enero de 2008). Plan Sectorial de Turismo 2007-2012. Diario Oficial de la PPA Federación. México: Autor.

Vamonos de Puente S.C. VDP, (2010). Análisis de sitios turísticos apoyados por la CDI. Documento informe cDI. 